\title{
HARMONIE-AROME, MODELO OPERATIVO DE ESCALA CONVECTIVA DE AEMET. PARTE I: MODELO DE PREDICCIÓN Y VALIDACIÓN
}

\author{
Javier Calvo, Daniel Martín, Gema Morales y Samuel Viana \\ AEMET, c/ Leonardo Prieto Castro s/n, 28040 Madrid \\ fcalvos@aemet.es
}

\section{Resumen}

Los modelos numéricos de la atmósfera son muy útiles para la predicción del tiempo a corto y medio plazo. La comunidad científica dentro del campo de la meteorología destina gran cantidad de recursos humanos y técnicos al desarrollo de los modelos de predicción numérica con el objetivo de lograr perfeccionar el pronóstico.

Estos modelos de predicción constituyen una representación físico-matemática de la atmósfera, complementados con el uso de las observaciones existentes en el momento de iniciar el pronóstico del estado futuro de la atmósfera.

Hoy día, las salidas de los modelos de predicción numérica del tiempo son utilizadas diariamente por parte de los predictores en los servicios meteorológicos. Por ello, es necesario conocer las características físicas y dinámicas en las que se basan estos modelos, para así, poder interpretar correctamente sus resultados.

Desde junio de 2017, HARMONIE-AROME es el nuevo modelo operativo de alta resolución de AEMET. Es un modelo no-hidrostático que resuelve la convección profunda explícitamente y que se integra con una resolución horizontal de $2,5 \mathrm{~km}$. Se presentan en estas jornadas de la AME las principales características del nuevo modelo.

\section{Introducción}

Los modelos de predicción numérica del tiempo han mejorado mucho en las últimas décadas debido a un gran aumento en la capacidad de cálculo que ha permitido utilizar operativamente modelos mucho más sofisticados y aumentar resolución. Los modelos globales siguen siendo hidrostáticos y se integran a resoluciones del orden de $10 \mathrm{~km}$. Los modelos de área limitada se han movido a escalas convectivas, del orden de $1 \mathrm{a} 4 \mathrm{~km}$, lo que requiere una dinámica no hidrostática y unas parametrizaciones físicas más complejas. Por otra parte, en estas escalas la incertidumbre es mayor y se suele recurrir a ensembles de integraciones para tratar de estimar la predecibilidad de la atmósfera. En la figura 1 se muestra la orografía comparada de dos modelos: global (ECMWF) y de área limitada (HARMONIE-AROME), para la islas Canarias.

$\mathrm{Al}$ sistema de predicción numérica del tiempo en AEMET se le conoce con el nombre de sistema HARMONIE-AROME. Constituye una configuración dentro del sistema compartido ALADIN-HIRLAM, fruto de la colaboración entre los consorcios ALADIN y HIRLAM. Formado por 26 servicios meteorológicos, se tiene previsto constituir un solo consorcio a finales de 2020. Otras configuraciones dentro del sistema ALADINHIRLAM son AROME, que se integra operativamente en Météo-France y ALARO, que utiliza una física especial que permite utilizarlos para las escalas grises de la convección 
(2-9 km). Todas estas configuraciones comparten gran parte de la dinámica y la asimilación con los modelo globales IFS y ARPEGE.

En este artículo se describen las características principales del modelo de predicción, su valor añadido comparado con otros modelos de menor resolución y se dan ejemplos de nuevos productos de predicción disponibles. La configuración HARMONIE-AROME está descrita en Bengtsson (2017) y está basada en el modelo AROME (Seity et al., 2011). La asimilación de datos constituye una parte muy importante de los modelos, sobre todo en las primeras horas de integración. El sistema de asimilación de HARMONIE-AROME es descrito en otro artículo de este volumen (Díez et al, 2018).

\section{Dinámica}

HARMONIE-AROME es un modelo No Hidrostático (Bubnova, 1995 y Benard, 2010), espectral, con una discretización semi-implícita semi-lagrangiana basadas en el esquema SETTLS (Hortal, 2002). La dinámica espectral es más precisa que la dinámica en puntos de grid y el esquema semi-lagrangiano permite pasos de tiempo largos (75 segundos para resoluciones de 2,5 km) con el consiguiente ahorro computacional. Por otra parte, en la vertical utiliza una coordenada híbrida y diferencias finitas y la proyección usada es la Lambert.

Una característica fundamental de este tipo de modelos no hidrostáticos es que los movimientos asociados con la convección profunda pueden ser resueltos (al menos parcialmente) por la dinámica del modelo. Hay muchas aproximaciones distintas para incluir los efectos no hidrostáticos. HARMONIE-AROME utiliza el esquema de Laprise (1992) que añade dos ecuaciones más de pronóstico (divergencia vertical del viento y la desviación de la presión), pero que permite seguir utilizando el código desarrollado para resolver el sistema de ecuaciones primitivas de los modelos hidrostáticos.
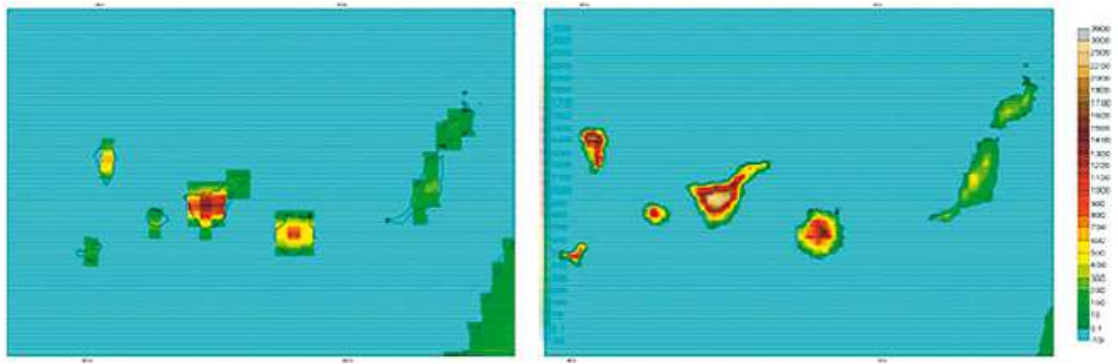

Figura 1: Comparación de la orografía utilizada por el modelo ECMWF (unos 16 km de resolución) y la utilizada por HARMONIE $(2,5 \mathrm{~km})$. El aumento de la resolución permite una mejor descripción de los efectos locales

\section{Parametrizaciones físicas}

Las parametrizaciones tratan de representar los procesos que ocurren a escalas no resueltas por la dinámica del modelo y los procesos que son demasiado complejos para 
ser tratados detalladamente. Hay que tener en cuenta que la resolución efectiva de un modelo puede ser unas 6 veces el espaciado de su rejilla, que para un modelo como HARMONIE sería de unos $15 \mathrm{~km}$. La física de HARMONIE-AROME está basada en la física del modelo de investigación MESO-NH (Bougeault et al., 2009) que ha sido adaptada para su utilización operativa.

El esquema de radiación es el del ciclo 32 del ECMWF. Se hace una separación entre la radiación de onda corta y la radiación de onda larga. La primera emplea 6 bandas del espectro y 16 bandas de la segunda utilizando valores climatológicos (constantes) para los aerosoles y los compuestos químicos. La representación de la radiación requiere muchos recursos de computación por lo que los flujos radiativos se actualizan cada 15 minutos. Los mayores errores en los cálculos de la radiación que llega a la superficie provienen de los errores en la predicción de las nubes y sus características. Otra fuente de error viene por no incluir a los aerosoles como variable de predicción.

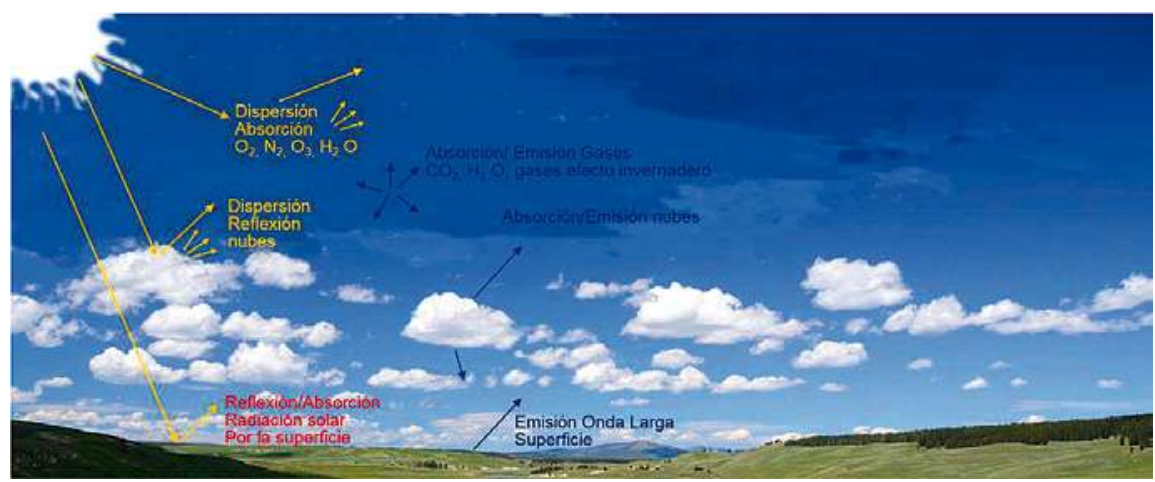

Figura 2: Parametrización de la radiación cuyos cálculos se dividen en onda larga y onda corta. Los mayores errores provienen de los errores en la descripción de las nubes

La parametrización de la turbulencia utiliza un esquema de energía cinética turbulenta (TKE) combinada con una longitud de mezcla de diagnóstico. El esquema original CBR (Cuxart et al., 2000) ha sido actualizado por HARATU (detalles en Bengtsson, 2017). Este esquema ha reducido la cobertura nubosa e incrementado la altura de la base de las nubes. También ha supuesto una considerable mejora en la climatología del viento del modelo.

La parametrización de la convección somera se trata mediante un esquema unificado denominado EDMF (Eddy-Diffusivity Mass-Flux). Este esquema considera que el flujo vertical turbulento es debido a dos aportaciones, por un lado la de los remolinos turbulentos (eddy-diffusivity) y, por otro, a la contribución de los ascensos convectivos de mayor escala (mass-flux). Los remolinos turbulentos son tratados mediante el esquema de turbulencia explicado anteriormente, mientras que para los ascensos convectivos se utiliza una aproximación de flujo de masa que, a diferencia de otros esquemas, es dual: considera dos tipos de ascensos, uno seco y otro húmedo. Las nubes asociadas con la convección somera son nubes sin precipitación pero tienen un gran 
impacto en la evolución de la capa límite y en la configuración de los entornos que dan lugar a convección profunda.

La parametrización de los procesos microfísicos, que determina los procesos de condensación y formación de la precipitación, es bastante compleja. El esquema de microfísica se denomina ICE3, porque usa tres clases de hielo (Pinty and Jabouille, 1998 y Lascaux et al., 2006). Esta parametrización refleja la interacción entre partículas acuosas en los distintos estados de la materia, que vienen representadas por seis de las variables del modelo: vapor de agua, agua de nube, cristales de hielo, lluvia, nieve y nieve granulada. Más de 25 procesos están parametrizados en este esquema, entre ellos la nucleación, la autoconversión, la sedimentación, la agregación, etc. Para mejorar la representación de las nubes a bajas temperaturas se han implementado en el esquema distintos desarrollos bajo la opción "OCND2 " que busca evitar la generación de partículas de hielo cuando se espera que aún exista agua subenfriada. Los hidrometeoros son transportados por advección mediante el esquema semi-lagrangiano y verticalmente mediante sedimentación. La fracción nubosa tridimensional se obtiene mediante un diagnóstico estadístico. Por otra parte, este esquema de microfísica hace una serie de suposiciones referentes a la distribución de tamaño de las partículas. Así, el diámetro de las especies acuosas responde a una función gamma generalizada, mientras que el tamaño de las gotas de lluvia sigue la ley exponencial de Marshall-Palmer.

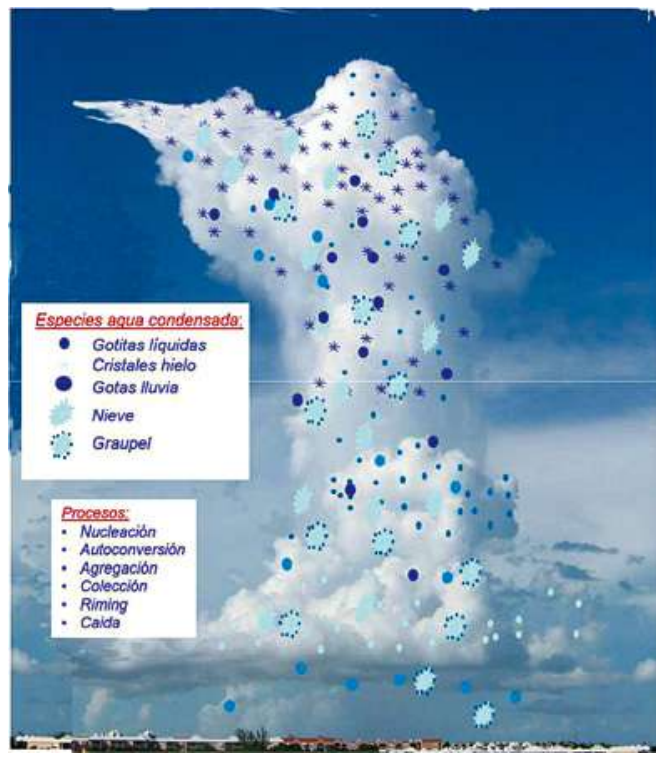

Figura 3: Parametrización de los procesos microfísicas que incluye 3 especies para el agua condensada y 3 para la precipitación

Los procesos superficiales se tratan mediante un modelo independiente denominado SURFEX (Masson et al., 2013). Para representar la heterogeneidad superficial se utiliza un esquema de tipo teselas que considera 4 tipos de superficie: suelos 'naturales', urbanos, lagos y océanos. Cada rejilla del modelo atmosférico es dividida en 4 teselas, cada una con una fracción y cuya evolución viene determinada por un modelo específico. Los suelos naturales utilizan el esquema ISBA (Interaction Soil Biosphere Atmosphere), también utilizado en el modelo HIRLAM, que distingue entre 12 tipos de vegetación. Es importante señalar que no se consideran intercambios horizontales entre las distintas teselas. Los flujos sobre mar se calculan utilizando el es- 
quema ECUME y para lagos se utiliza un modelo sencillo 1D denominado FLAKE. Para la evolución de la nieve se utiliza un modelo sencillo de una sola capa (Douville, 1995) que permite predecir el espesor de la nieve. Para suelos urbanos se utiliza un modelo específico denominado Town Energy Budget (TEB). Los esquemas de superficie dependen mucho de la calidad de los datos fisiográficos (tipos de suelo, uso, distribución vegetal, etc.). La base de datos fisiográfica utilizada es ECOCLIMAPv2.2, (Masson et al, 2003) mientras que la topografía se basa en GMTED2010 con 250 m de resolución.

\section{Integración operativa en AEMET}

La integración de HARMONIE-AROME requiere 12 veces más potencia de cálculo que el modelo HIRLAM. Su integración operativa ha sido posible gracias al superordenador Bullx instalado en AEMET que tiene una potencia pico de cálculo de 168 Teraflops. Desde junio de 2017 el modelo ha sido declarado como el modelo operativo sustituyendo a HIRLAM. La versión 40h1.1 del modelo HARMONIE-AROME se ejecuta a una resolución horizontal de $2,5 \mathrm{~km}$, realizándose ocho pasadas al día a intervalos de 3 horas. Se ejecuta en dos dominios diferentes (fig. 4), uno que cubre la península ibérica y las islas Baleares y un segundo para las islas Canarias. El alcance de las predicciones es de 48 horas con salidas para una selección de variables cada 15 minutos.

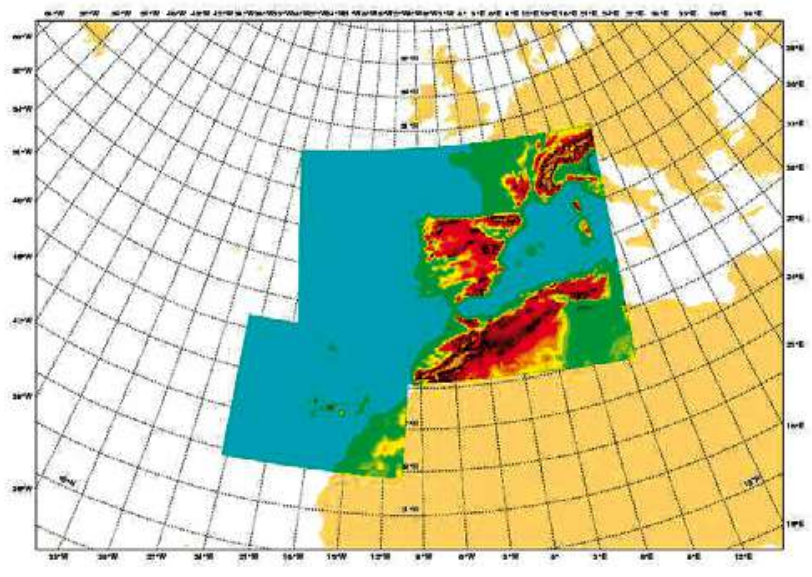

Figura 4: Dominios operativos de integración del modelo HARMONIEAROME

Las condiciones de contorno provienen de la predicción del modelo del ECMWF correspondientes a un ciclo con 6 horas de antigüedad. Las predicciones a 48 horas están disponibles unas 2 horas 40 minutos después de la hora nominal del ciclo.

\section{Validación y verificación}

Una forma inmediata de comprobar que un modelo está dando buenas predicciones es comparar a lo largo del tiempo la predicción de una variable en un punto con los valores observados en ese mismo lugar. En la fig. 5 se comparan las predicciones de la temperatura a $2 \mathrm{~m}$ durante el mes de junio de 2017 en el aeropuerto de Madrid-Barajas proporcionadas por los modelos HIRLAM y HARMONIE-AROME comparadas con los valores observados. La mejora que supone HARMONIE-AROME es clara, mostrando HIRLAM un claro sesgo negativo. 


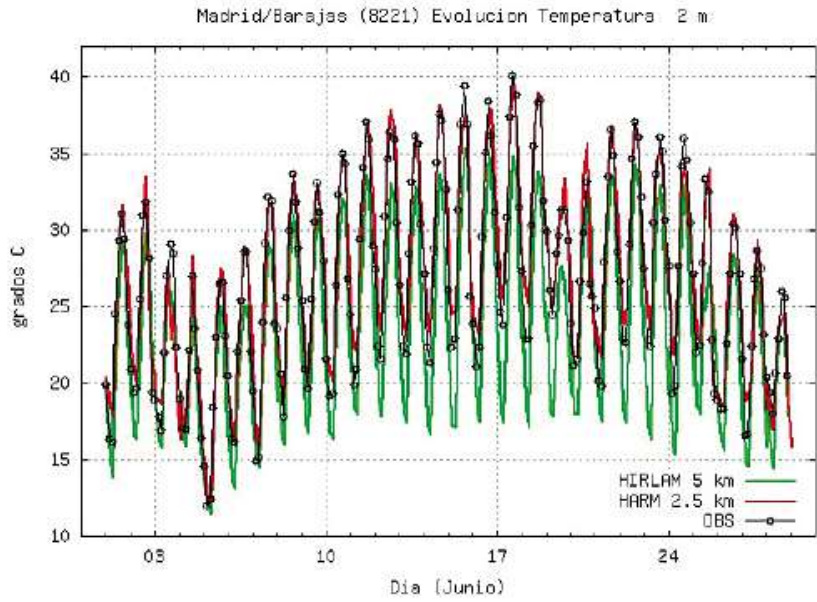

Figura 5: Predicciones de la temperatura a $2 \mathrm{~m}$ en Madrid-Barajas durante el mes de junio de 2017 comparadas con las observaciones (línea negra). La mejora de HARMONIE (línea roja) respecto a HIRLAM (línea verde).

A fin de comprobar de una manera objetiva que un modelo nuevo está dando mejores predicciones que otros, lo que se hace es calcular una serie de estadísticos a partir de una muestra la suficientemente significativa de valores observados frente a valores predichos. A este proceso se denomina verificación. Los parámetros estadísticos clásicos son el sesgo, o bias, y la desviación estándar. Usando los valores de estos parámetros para la temperatura y el viento a $10 \mathrm{~m}$, se ha comprobado la mejora esperada (no se muestra).

También se suele recurrir a una verificación categórica calculando estadísticos para distintos rangos de predicción. Este método se aplica por ejemplo para la precipitación como se muestra en la fig. 6. La mejora de HARMONIE-AROME respecto a HIRLAM es clara mientras que HARMONIE-AROME muestra valores similares de ETS que ECMWF. Hay que tener en cuenta que este tipo de estadísticos puntuales penalizan a los modelos con mayor resolución (variabilidad) debido al efecto de doble-penalización, siendo más adecuado utilizar métodos de verificación espacial para comparar modelos de distintas resoluciones.

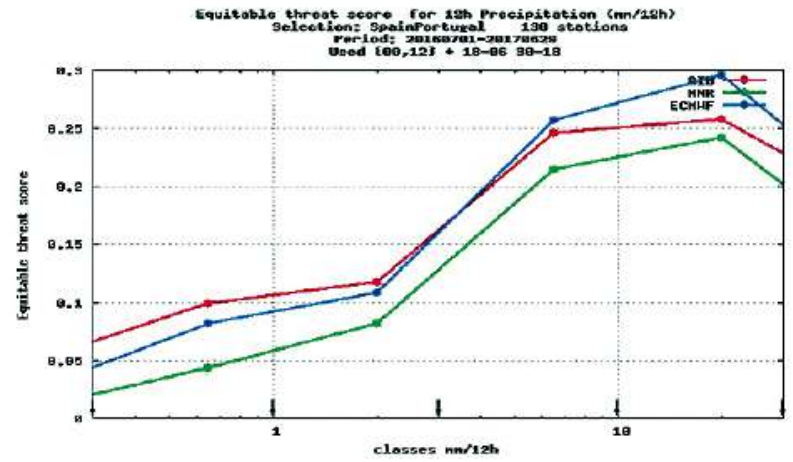

Figura 6: Verificación categórica de la precipitación para distintas intensidades. Se muestra el Equitable Threat Score (ETS) calculado para todas las predicciones durante un año. Valores mayores del ETS significan menor error. Se comparan HARMONIE (roja), HIRLAM $5 \mathrm{~km}$ (verde) y ECMWF (azul). 
Las nuevas variables de predicción del modelo han permitido el desarrollo de nuevos diagnósticos muy útiles para la validación del modelo y como herramientas de predicción. Así las descargas eléctricas se estiman a partir del graupel integrado en la vertical y se ha visto que esta estimación correlaciona bien con las observaciones de la red de descarga de AEMET. También se pueden simular pseudo-imágenes radar o de distintos canales de satélite como puede verse en la figura 7.
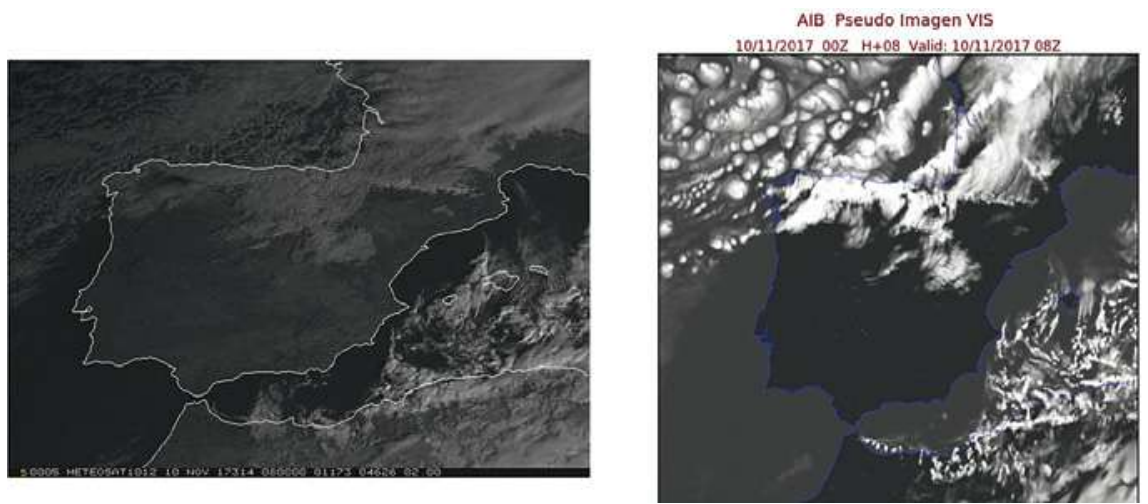

Figura 7: Imagen visible del satélite METEOSAT para el día 10/11/2017 a las 08 UTC comparada con una predicción del modelo HARMONIE.

Por otra parte, HARMONIE-AROME mejora significativamente la predicción de nubes bajas y nieblas aunque estas siguen siendo un fenómeno difícil de predecir. Probablemente el mayor valor añadido de HARMONIE está en la predicción de precipitación. Es capaz de reproducir intensidades mayores que los modelos hidrostáticos que necesitan parametrizar la convección, reproduce mejor el ciclo diurno de la convección y, al tener mayor resolución de la orografía, simula mejor los efectos locales. En la figura 8 puede verse un ejemplo de imagen radar simulada con HARMONIE comparada con la
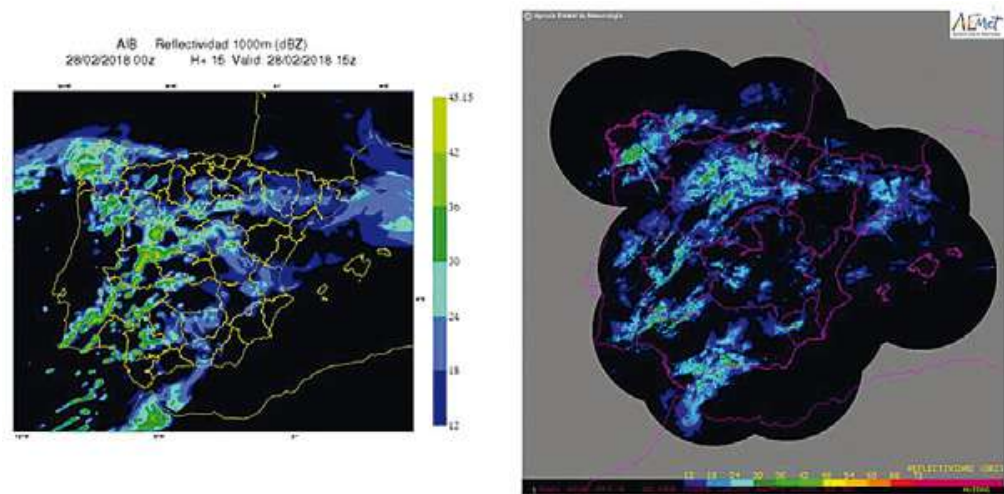

Figura 8:

Imagen radar para el 28/02/2018 comparada con la pseudoimagen predicha por HARMONIE. 
observación. Puede verse que las estructuras son bastante realistas aunque con errores locales y temporales debido a la incertidumbre asociada a los fenómenos convectivos.

\section{Conclusiones}

El modelo HARMONIE-AROME es oficialmente el modelo operativo de AEMET desde junio de 2017. Es un modelo no hidrostático que resuelve explícitamente la convección y que ha supuesto un gran avance en la predicción determinista en AEMET. En este artículo se han resumido las características más importantes del modelo de predicción mientras que en Díez et al (2018), también en este volumen, se describe la asimilación de datos realizada por el modelo. El modelo forma parte del llamado sistema compartido ALADIN-HIRLAM, fruto de la colaboración entre los consorcios ALADIN y HIRLAM. Por otra parte, la modelización de alta resolución requiere grandes recursos de cálculo por lo que su implementación operativa sólo ha sido posible con la instalación del sistema de supercomputación Bullx con un rendimiento pico de 168 Teraflops.

El nuevo modelo ha supuesto una mejora significativa en la predicción de fenómenos locales como viento y temperatura pero, probablemente donde ha supuesto una mayor mejora es en la predicción de nieblas y nubes bajas, y en la predicción de la precipitación.

No obstante, la modelización a estas escalas supone un cambio de paradigma respecto a los modelos hidrostáticos: la variabilidad y la incertidumbre asociada a las mismas es mucho mayor y hay que recurrir a interpretaciones probabilísticas de las predicciones.

\section{Referencias}

- Bénard, P., Vivoda, J., Maek, J., Smolíková, P., Yessad, K., Smith, C., Broková, R., Geleyn, J., 2010: Dynamical kernel of the Aladin-NH spectral limited-area model: Revised formulation and sensitivity experiments. Quarterly Journal of the Royal Meteorological Society 136(646), 155-169.

- Bengtsson, L., Andrae, U., Aspelien, T., Batrak, Y., Calvo, J., et al, 2017: The HARMONIE-AROME Model Configuration in the ALADIN-HIRLAM NWP System. Monthly Weather Review 145, N. ${ }^{\circ}$ 5, 1919-1935.

- Bubnová, R., Gwenäelle, H., Bénard, P., Geleyn, J, 1995: Integration of the fully elastic equations cast in the hydrostatic pressure terrain-following coordinate in the framework of the ARPEGE/ Aladin NWP System. Monthly Weather Review 123(2), 515-535.

- Bougeault, P., Mascart, P., et al., 2009: The Meso-NH atmospheric simulation system: Scientific Documentation part iii: Physics. Tech. rep.

- URL http://mesonh.aero.obsmip.fr/mesonh/dirdoc/book1m4819jan2009/scidocp3.pdf

- Cuxart, J., P. Bougeault, and J.-L. Redelsberger, 2000: A turbulence scheme allowing for mesoscale and large-eddy simulations. Quart. J. Roy. Meteor. Soc., 126, 1-30.

- Diez, M., 2018: HARMONIE-AROME, modelo operativo de escala convectiva de AEMET. Parte II: Asimilación de datos. XXXV Jornadas Científicas de la AME, 5-7 marzo 2018, León.

- Douville, H., Royer, J., Mahfouf, J., 1995: A new snow parameterization for the Météo-France climate model. Climate Dynamics 12(1), 21-35.

- Hortal, M.202: The development and testing of a new two-time-level semi-lagrangian scheme (SETTLS) in the ECMWF forecast model. Quart. J. Roy. Meteor. Soc., 128(583), 1671-1687. 
Laprise, R., 1992: The euler equations of motion with hydrostatic pressure as an independent variable. Monthly Weather Review 120(1), 197-207.

- Lascaux, F., E. Richard, adn J.-P. Pinty, 2006: Numerical simulations of three map IOPs and the associated microphysical processes. Quart. J. Roy. Meteor. Soc., 132, 1907-1926.

- Masson, V., Champeaux, J.L., Chauvin, F., Meriguet, C., Lacaze, R., 203: A global database of land surface parameters at $1-\mathrm{km}$ resolution in meteorological and climate models. Journal of Climate 16(9), 1261-1282.

- Masson, V., Le Moigne, P., Martin, E., Faroux,„S. et al., 2013: The surfexv7.2 land and ocean surface platform for coupled or offline simulation of earth surface variables and fluxes. Geoscientific Model Development 6(4), 929-960 (2013). DOI 10.5194/gmd-6 -929-2013. URL https://www.geoscimodel-dev.net/6/929/2013/

- Pinty, J.-P., and P. Jabouille, 1998: A mixed-phase cloud parameterization for use in a mesoscale non-hydrostatic model: Simulations of a squall line and of orographic precipitation. Preprints, Conf. on Cloud Physics. Everett, WA, Amer. Meteor. Soc., 217-220.

- Seity, Y., Brousseau, P., Malardel, S., Hello, G., Bénard, P., Bouttier, F., Lac, C., Masson, V., 2011: The AROME-France convective-scale operational model . Monthly Weather Review 139(3), 976-991. 